\title{
ZIGOMICOSIS CUTANEA PRIMARIA POR Rhyzopus oryzae EN UNA NIÑA CON LEUCEMIA LINFOBLASTICAAGUDATIPO B
}

\author{
(Primary Cutaneous Zygomycosis by Rhyzopus oryzae in a girl diagnosed \\ with an Acute Lymphoblastic Leukemia B Type)
}

\author{
R. Salim (1), R. Runco (1, 2), C. Alvarez (2), S. Romano (3) \& M. Charre (2) \\ 1. Cátedra de Micología-Fac. de Bioquímica, Química y Farmacia \\ Universidad Nacional de Tucumán Ayacucho 491. (4000) Tucumán. R. Argentina. \\ 2. Lab. Micología del Hospital del Niño Jesús. Pasaje Hungría 750.(4000) Tucumán. R. Argentina. \\ 3. Cátedra de Dermatología- Fac. de Medicina. U. N. T. \\ Ayacucho 491. (4000). R. Argentina. \\ e-mail: rqsalim@rectorado.unt.edu.ar
}

Palabras claves: zigomicosis cutánea primaria, leucemia, Rhizopus oryzae Key words:Primary cutaneous zygomycosis, leukemia, Rhizopus oryzae

\section{RESUMEN}

La Zigomicosis es una infección infrecuente causada por hongos oportunistas integrantes del orden Mucorales, que se presenta en pacientes de alto riego como en: leucemia, linfomas con neutropenia prolongada, cetoacidosis diabética, malnutrición severa, ruptura de la integridad de la barrera cutánea y terapia inmunosupresora. Se presenta un caso de Zigomicosis cutánea en una paciente pediátrica con leucemia linfoblástica aguda de tipo $B$, con severa neutropenia y tratamiento con corticoides. A los cinco días de su hospitalización desarrolló en el antebrazo (zona de punción venosa), una lesión indurada, eritematosa, que progresó y ulceró. A partir de exudados y biopsias del tejido subcu-táneo se realizaron exámenes microscópicos directos con $\mathrm{KOH}$, cultivos en agar Sabouraud y estudio histológico a través de técnicas convencionales de hematoxilina-eosina y PAS. Los análisis de los materiales clínicos revelaron la presencia de hifas hialinas, no tabicadas, gruesas, compatibles con un Zygomycete. En todos los tubos se obtuvo abundante desarrollo de un hongo filamentoso, identificado como Rhizopus oryzae. Posteriormente a la escisión quirúrgica y tratamiento con anfotericina $B$ se obtuvo una evolución favorable del paciente hasta el presente.

\section{INTRODUCCION}

Los hongos del orden Mucorales son los agentes causantes de lo que clásicamente llamamos mucormicosis

Recibido el 12 de Abril 2008

Aceptado el 8 de Octubre 2008

\section{ABSTRACT}

Zygomycosis is an infrequent infection caused by opportunistic fungi which belong to the order Mucorales and which is present in high risk patients diagnosed with : leukemia, lymphomas with prolonged neutropenia, diabetic cetoacidosis, severe malnutrition, rupture of the entire cutaneous barrier and immunesuppressing therapy. This paper deals with a case of cutaneous Zygomycosis in a pediatric patient diagnosed with acute lymphoblastic leukemia B type, suffering a severe neutropenia and corticosteroid treatment. On the fifth day of hospitalization, her forearm (venous puncture zone) showed an indured, erimatose lesion which progressed and ulcerated. Collection of exudates and biopsies of subcutaneous tissue served to carry out direct microsco-pic examinations with $\mathrm{KOH}$, cultures in Sabouraud Agar and a histologic study through conventional hematoxilin-eosin and PAS techniques. Analyses of the clinical materials revealed the presence of hyaline, not septated and broad hyphae suitable to a Zygomycete. In all the tubes there was an abundant development of filamentous fungus identified as Rhizopus oryzae. After the surgical scission and treatment with anfotericine $B$, the patient showed a favorable evolution up to now.

o zigomicosis. Los géneros y especies de la familia Mucoraceae, son los que causan más frecuentemente zigomicosis y de ellos los más comunes son Rhizopus oryzae y $\boldsymbol{R}$. microsporus var. rhizopodiformis. Otros agentes etiológicos descritos en el hombre son: Rhizomucor pusillus, Absidia corymbifera, Cunninghamella 
bertholletiae y con menor frecuencia se diagnostican casos originados ocasionados por Saksenaea vasiformis, Mucor circinelloides, M. ramosissimus, Apophysomyces elegans, Cokeromyces recurvatus y Syncephalastrum racemosum (20, 21, 23, 29, 32). En pacientes pediátricos, los agentes etiológicos identificados han sido fundamentalmente Rhizopus spp. y esporádicamente Absidia spp. $(1,20,23,28,37)$.

Los Mucorales no forman parte de la microbiota residente del hombre. Ocasionalmente es posible encontrarlos como agentes saprobios de la piel y mucosas de individuos sanos o de pacientes hospitalizados (14, 21, 22, 35). Esta cualidad, junto con su facilidad para crecer en los medios habituales para hongos, hace que los cultivos positivos constituyan, en sí mismos, tan sólo una sospecha de infección, y que ésta deba ser confirmada por la demostración de los hongos en los tejidos afectados $(8,28)$.

La característica epidemiológica más relevante de los Zygomycetes es su ubicuidad. Han sido identificados en el medio ambiente hospitalario e incluso en los sistemas de aire acondicionado, lo que representa, al igual que Aspergillus spp., una amenaza para la población de pacientes inmunocomprometidos $(35,36)$. Las esporangiosporas pueden ser vehiculizadas en el ámbito hospitalario por corrientes de aire o por objetos en contacto directo con los pacientes (gasas, vendas, una variedad de productos adhesivos usados en su ajuste y bajalenguas de madera contaminados), que pueden originar infecciones nosocomiales (7, 9, 12, 22, 25, 32).

La infección se adquiere principalmente por inhalación de esporas, las que colonizan los senos paranasales y nasofaringe; ocasionalmente se puede adquirir por inoculación cutánea de esporas o ingestión inadvertida de productos contaminados. No se ha documentado transmisión de persona a persona (14).

Los factores que predisponen la infección por estos hongos son múltiples. Entre ellos: acidosis metabólica, cetoacidosis diabética, el uso de Desferoxamina, leucemia en fase de neutropenia prolongada, linfoma y síndrome mielodisplásico, trasplante de órganos sólidos y de precursores hematopoyéticos, desnutrición severa, ruptura de la integridad de la barrera cutánea (cirugía, traumatismos, quemaduras graves, punciones con agujas contaminadas, mordeduras o picaduras de insectos, vendajes contaminados, inserción de catéter, pinchazos y otros tipos de lesiones cutáneas, etc.) $(12,13,17,23,25$, 26, 29 ,31, 37). También pueden afectar a enfermos bajo terapéuticas inmunosupresoras, niños prematuros y a infectados por $\operatorname{VIH}(1,8,13,17,18,19,32,34,38)$.

Después del contacto con los tejidos, estos hongos raramente producen infecciones e invasión, y en una alta proporción de los casos se comportan como meros colonizadores (32). La integridad de las barreras cutáneomucosas y un sistema inmunitario competente son las primeras líneas de defensa con que se encuentran las esporas.

La enfermedad se caracteriza por la rápida invasión de tejidos y estructuras vasculares con las consiguientes vasculitis y trombosis que conducen a infartos y necrosis tisular (2, 3, 5, 7, 12, 14, 15, 22, 24, 25, 27, 28, 31, 32, 38). Típicamente, la infección implica el área rhino-facial-craneal, los pulmones, el tracto gastrointestinal, la piel, y menos comúnmente otros sistemas de órganos $(21,22)$.

Se describen seis formas clínicas de mucormicosis: cutánea, gastrointestinal, pulmonar, rinocerebral, diseminada y una forma miscelánea, que engloba localizaciones como endocarditis, miocarditis, meningitis, absceso cerebral, endoftalmitis, cistitis, peritonitis, osteomielitis, artritis, miositis y pielonefritis (14, 20, 29, 32). En niños se han descrito principalmente las formas cutánea e intestinal en neonatos y las formas pulmonares y rinocerebral en pacientes diabéticos e inmunocomprometidos (1, 17, 37, 38).

La zigomicosis cutánea primaria se clasifica en: superficial, nodular, y gangrenosa. En cualquier caso, se inicia como vesículas o pústulas que pronto se transforman en úlceras o escaras.

El tipo superficial es poco sintomático y tiene el aspecto de una úlcera que no sana. El tipo gangrenoso muestra una progresión fulminante con extensión hacia los tejidos profundos. Otras formas incluyen púrpura, induración y ulceración con formación de escara. En la periferia de las lesiones cutáneas, generalmente existen áreas de celulitis que pueden ser intensamente dolorosas a la palpación, aunque algunas zonas pueden ser insensibles debido a la necrosis (19). Pueden ser rápidamente agresivas, incluso frente al desbridamiento apropiado y al tratamiento médico (32).

En el huésped immunocompetente, se manifiesta con vesículas o pústulas que eventualmente progresan formando costras (12). Por el contrario, en el paciente inmunocomprometido las lesiones, rojas e induradas, progresan formando costras negras que, al desprenderse, dejan úlceras grandes. La preferencia angioinvasiva de los zygomycetes, con la consecuente diseminación por debajo de las estructuras cutáneas, culmina con la necrosis rápida y progresiva no sólo de los tejidos cutáneos y subcutáneos, sino también de la grasa, músculos, tendones, el hueso adyacente y seguir un curso fulminante $(3,12,15,23,26,30,31)$.

Frente a este tipo de lesiones cutáneas, se debe realizar diagnóstico diferencial con infecciones bacterianas necrotizantes, aspergilosis, tuberculosis, sífilis y otros procesos granulomatosos. El diagnóstico de zigomicosis 
se establece por la demostración de las hifas en el material de biopsia.

En este trabajo describimos los aspectos histológicos y clínicos de una zigomicosis cutánea primaria causada por Rhizopus oryzae en el antebrazo de una paciente pediátrica con leucemia linfoblástica aguda de tipo $\mathrm{B}$, con severa neutropenia y tratamiento con inmunosupresores.

\section{CASO CLINICO}

Paciente femenina, de 2 años de edad, residente en Alto Verde, Concepción, (Tucumán - R. Argentina) consulta por cuadro clínico de 15 días de evolución, con lesión ampollar en la región submaxilar izquierda, tos y fiebre persistente. Es medicada con Cefalexina (140/mg/ kg/día) durante 9 días. Debido a la mala evolución de la lesión, fiebre persistente y disminución de peso, se decide su internación en el Hospital de Concepción. El día 20 de febrero de 2008 ingresa febril, con palidez mucocutánea, poliadenopatía latero-cervical, supraclavicular izquierda, axilar e inguinal y hepatoesplenomegalia. Es tratada con Clindamicina y Dipirona. Después de recibir los resultados de laboratorio se diagnostica pancitopenia febril. Es derivada con urgencia al Hospital del Niño Jesús el 22/2/ 2008. A su ingreso impresiona como una paciente en estado general grave, hemodinámicamente compensada, con síndrome linfoproliferativo y absceso submaxilar izquierdo de etiología desconocida.

Con diagnóstico presuntivo de leucemia, es derivada a la Sala de Inmunodeprimidos. Presenta poliadenopatias móviles y dolorosas a la palpación, petequias en los miembros inferiores y tumefacción eritematosa en región submaxilar izquierda. Es medicada con Ceftazidima, Amikacina y Clindamicina. Sus exámenes

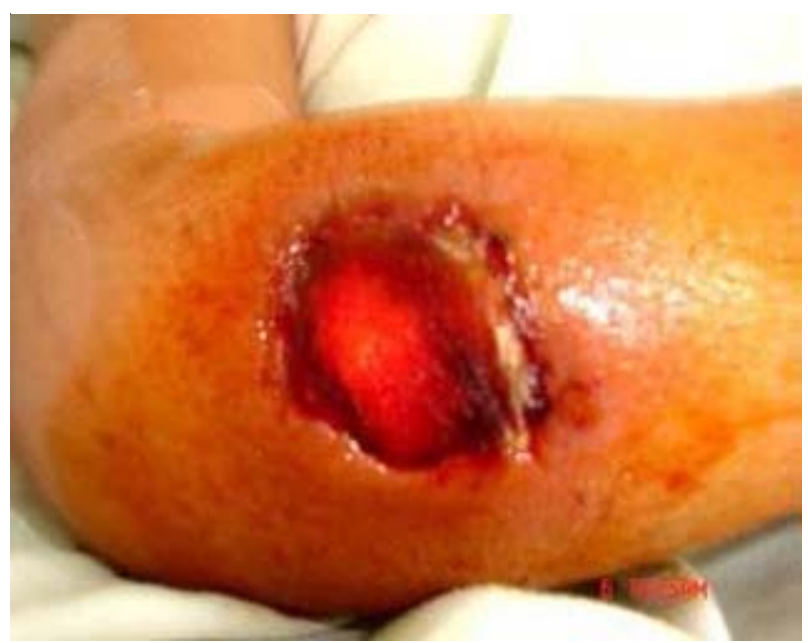

Fig. 1. Zigomicosis cutánea primaria con necrosis

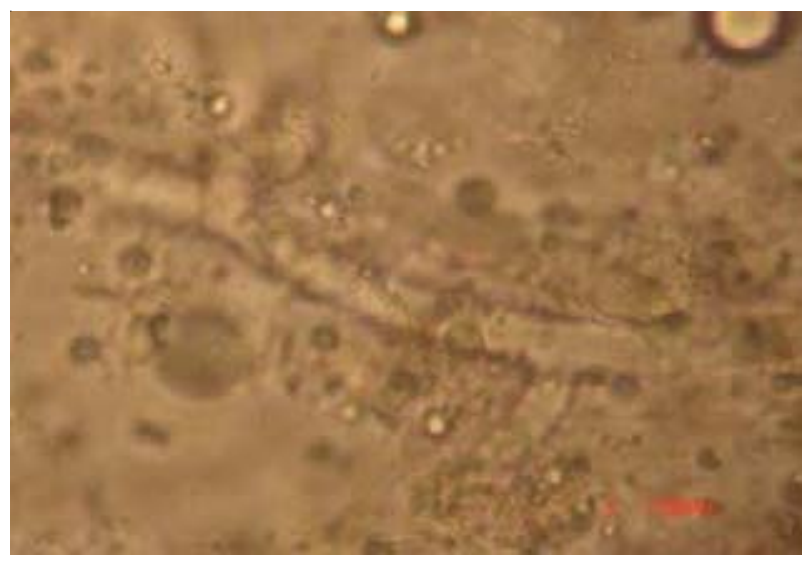

Fig. 2. Hifas cenocíticas en el examen directo (KOH $10 \%)$ de muestra de exudado seropurulento

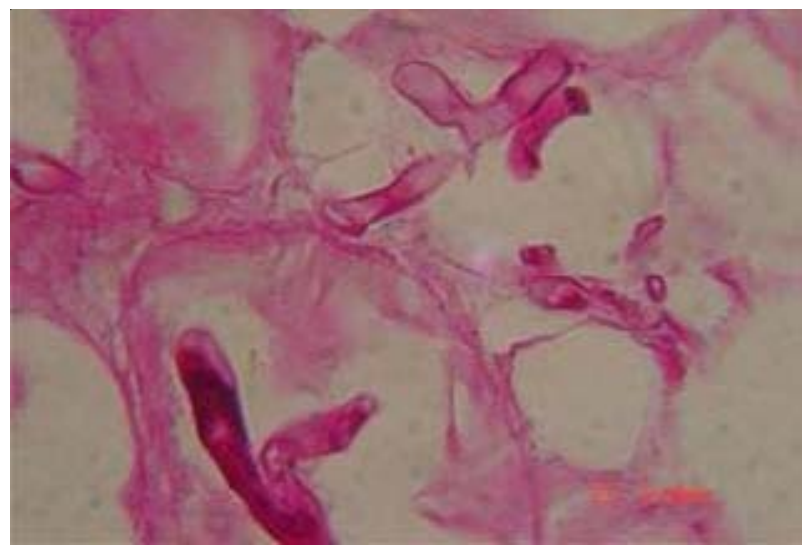

Fig. 3. Hifas anchas no tabicadas, Coloración HE (100X)

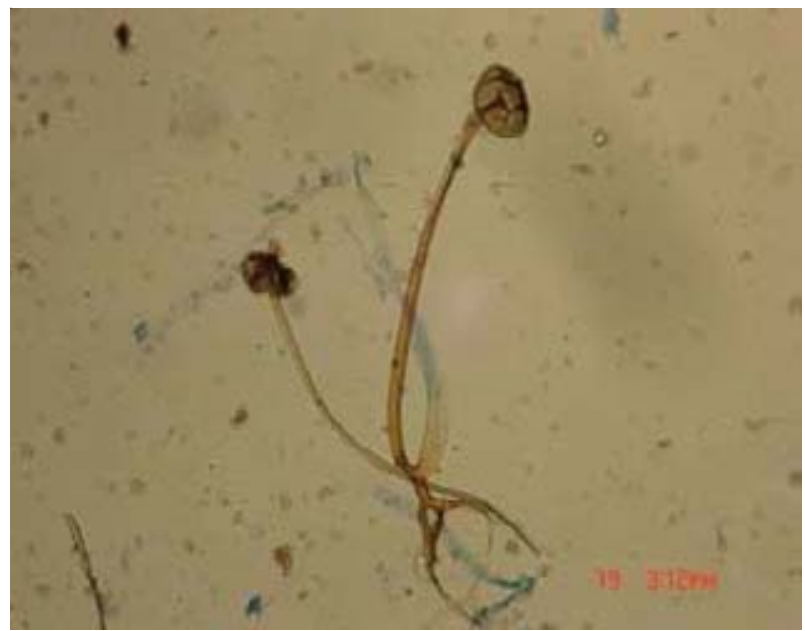

Fig. 4. Aspecto micromofológico (Gueguén 40X)

de laboratorio iniciales mostraron: leucocitos 25.000 cel; células blásticas $97 \%$, linfocitos $2 \%$, monocitos $1 \%$, hemoglobina $6,20 \mathrm{~g} / \mathrm{dl}$, hematocrito $19,6 \%$ y plaquetas 18000. Se observa marcada hipocromía y anisocromía. glicemia normal; proteínas totales $8.6 \mathrm{~g} / \mathrm{dl}$, urea $0.42 \mathrm{~g} / \mathrm{L}$; 
GPT 45 U/L. El laboratorio de Microbiología informó hemocultivo negativo, $\boldsymbol{E}$. coli en urocultivo y Staphylococcus aureus en el exudado del absceso submaxilar. Al examen micológico directo del exudado no se observaron elementos fúngicos ni se aislaron hongos en los medios de cultivo específicos.

A los cinco días de su hospitalización (27/2/08), desarrolló en el antebrazo derecho, correspondiendo a la zona de punción venosa, una lesión indurada, eritematosa, que progresó y en cinco días se ulceró (3/3/08). La lesión, de 7 por $5 \mathrm{~cm}$, dolorosa, tórpida, irregular, de bordes policíclicos e hipertróficos, centralmente necrótica, rodeada de piel eritematosa que presentaba un fondo irregular con exudado seropurulento (Fig. 1). Se realizó toma de muestra por punción aspiración de la lesión y sangre que se enviaron para examen bacteriológico y micológico (directo y cultivos). Los hemocultivos fueron negativos. Los cultivos bacterianos fueron positivos para S. aureus meticilino resistente. Al examen micológico directo (KOH, Giemsa y Gram) se observaron hifas hialinas cenocíticas semejantes a Zygomycetes (Mucorales) resultado que fue inmediatamente informado al médico tratante (Fig.2). Se inició tratamiento con antibióticos parenterales (Ceftazidima y Amikacina), sin presentar mejoría. A las $72 \mathrm{~h}$ de incubación a $37^{\circ} \mathrm{C}$ en los cultivos en Agar Sabouraud Glucosa (SGA), se obtuvo el desarrollo de colonias lanosas y blanquecinas de un hongo filamentoso, hialino, con hifas cenocíticas, compatible con Rhizopus sp. Respecto a este resultado se mantuvo conducta expectante.

Dos días después (5/3/08), se recibió muestra de una escarectomía de la lesión necrótica para análisis microbiológico. Al examen micológico directo nuevamente se observaron hifas hialinas cenocíticas. En los cultivos en SGA a las $72 \mathrm{~h}$ de incubación a $37^{\circ} \mathrm{C}$ se obtuvo, otra vez, el desarrollo de colonias lanosas y blanquecinas de un hongo filamentoso, hialino, con hifas cenocíticas, posteriormente identificado como Rhizopus oryzae (Fig. 3 y 4). Se informaron los cultivos de hongos como positivos y ante el diagnóstico presuntivo de zigomicosis, el día 6/ 3/08 se realizó exéresis quirúrgica amplia y se inició medicación con Anfotericina B liposomal (1,5mg/Kg/dia) por vías intravenosa y tópica, más limpiezas quirúrgicas cada 3-4 días manteniendo terapia antibacteriana. El día 13/03/08 se recibió nuevo material de tejido subcutáneo necrótico y músculo para estudios micológicos e histopatológicos. El examen directo y cultivos de la muestra de músculo fueron negativos mientras que el análisis microscópico del tejido subcutáneo volvió a revelar hifas idénticas a las muestras anteriores. Nuevamente se obtuvo cultivo puro de $\boldsymbol{R}$. oryzae, en todos los cultivos del tejido subcutáneo.

El estudio histopatológico, a través de técnicas convencionales de hematoxilina-eosina y PAS, reveló extensas áreas de necrosis en dermis e hipodermis con presencia de abundantes hifas no septadas y anchas penetrando la dermis y los vasos sanguíneos, confirmando el diagnóstico micológico (Fig. 2).

Como complemento de la terapia antifúngica, la zona fue lavada en reiteradas oportunidades con solución salina fisiológica estéril y tratamiento topico con el antifúngico, repitiéndose los aseos quirúrgicos. Se procedió a la ampliación quirúrgica y repetición de las limpiezas curativas cuando se consideró necesario.

Con diagnóstico final de Leucemia Linfoblástica Aguda Tipo BII y zigomicosis cutánea primaria, a la fecha, la paciente presenta una evolución ligeramente favorable y continúa con quimioterapia específica para LLA, tratamiento antifúngico con Anfotericina B y aseos quirúrgicos periódicos. Por su pronóstico reservado, se evalúa la posibilidad de su traslado a Buenos Aires.

\section{Estudio Micológico}

Al examen microscópico directo de las muestras con $\mathrm{KOH}$ al $10 \%$, coloración de Gram y Giemsa, se observaron abundantes hifas hialinas, no septadas, anchas como cintas, de 10-20 $\mu \mathrm{m}$ de diámetro, con ramificaciones en ángulo recto.

Las muestras fueron sembradas en Agar Sabouraud Glucosa (SGA) adicionado de Penicilina (50 U.I./ml) y Estreptomicina $(80 \mu \mathrm{g} / \mathrm{ml})$, e incubadas a $37^{\circ} \mathrm{C}$. El diagnóstico microbiológico se basó en la observación macro y micromorfológica de las colonias en SGA. A partir de las 72 h se observó en todos los tubos abundante desarrollo de un hongo de crecimiento rápido. Las colonias cubrieron prácticamente toda la superficie del medio de cultivo, con micelio aéreo denso, algodonosas, de aspecto consistente, al principio blancas, después gris oscuras que se fueron ennegreciendo con el transcurso de los días, anverso claro, típicas de los integrantes del género Rhizopus (Fig.3).

Para la observación macro y micromorfológica se resembró la cepa en Agar Malta siguiendo la metodología de Schipper (27). Colonias café grisáceas, esporangióforos café-grisaceos, rizoides cafesosos, esporangioforos y estolones hasta $1200 \mu \mathrm{m}$ de largo y de 9-15 $\mu \mathrm{m}$ de ancho, solitarios o agregados en grupo, frecuentemente ramificados. Esporangios cafesosos a negros, globosos a subglobosos, lisos a finamente espinosos, 55-130 $\mu \mathrm{m}$; columelas elipsoidales a ovoides de base trunca, 40-100 $\mu \mathrm{m}$ de alto, de color gris; esporangiosporas esféricas, ovoides o irregulares, estriadas, pálidas, 4-8 $\mu \mathrm{m}$ de largo. Clamidosporas escasas, globosas a elipsoidales. Zigosporas ausentes. Crecimiento óptimo a $37^{\circ} \mathrm{C}$, máximo $42-43^{\circ} \mathrm{C}$. No crece a $45^{\circ} \mathrm{C}$. Determinación final: Rhizopus oryzae (Fig. 4). 


\section{DISCUSION}

La presentación clínica de la zigomicosis cutánea es variable, sin embargo, los signos clave que deben advertir al clínico son la necrosis y la rapidez del curso evolutivo (25). Una herida en cuyo margen aparece una necrosis progresiva en un paciente con factores de riesgo, debe orientar hacia zigomicosis. La triada constituida por necrosis cutánea, progresión veloz del cuadro, junto con los factores de riesgo del paciente, debe alertar al médico sobre un cuadro grave. La sospecha clínica de esta entidad, su estudio mediante examen directo de la escara, y el procesamiento rápido del material de biopsia para estudio convencional y cultivo, son determinantes para instaurar el tratamiento con la mayor celeridad posible, lo que redundará en un mejor pronóstico de esta grave enfermedad (8).

Los Zygomycetes esporádicamente forman parte de la microbiota saprofita normal de la piel y mucosas y crecen fácil y rápidamente en los medios de cultivo (22). Por esto, se explica que su diagnóstico no se puede establecer sólo por la positividad de los cultivos, sino que resulta indispensable demostrar la invasión fúngica en los tejidos, para lo que se requieren procedimientos agresivos como la obtención de biopsia de tejidos $(5,22)$. La invasión del tejido por hongos no tabicados mostrando áreas de necrosis e infartos con vasculitis, trombosis y hemorragias características de esta enfermedad constituye la prueba determinante de esta micosis $(25,28,29)$, y su cultivo es definitivo para la identificación final del agente (8).

A pesar de que el diagnóstico de la zigomicosis se basa en exámenes histopatológicos, es necesario tener en cuenta que éstos no suelen estar disponibles en forma rápida. Esta limitación justifica que, en algunas series, hasta el 50\% de los casos sólo fueron diagnosticados post mortem $(16,18)$. Por ello, el examen micológico directo de la escara es de gran rendimiento diagnóstico y en sólo unos minutos orientará hacia esta posibilidad.

En un estudio que reunió datos de 50 hospitales de España, el aislamiento de Zygomycetes fue muy bajo, demostrando que menos del $8 \%$ de los pacientes con cultivo positivo estaban realmente infectados, mientras que la gran mayoría presentaban colonización o contaminación de laboratorio (33). Aún así, consideramos que, si bien la trascendencia del cultivo puede ser baja, el aislamiento de estos hongos tiene importancia si se considera el tipo de lesión y procedencia de la muestra clínica. La observación de abundantes hifas hialinas no tabicadas junto al aislamiento de un zygomycete debe ser la voz de alarma para el seguimiento de los pacientes de riesgo, aun cuando su carácter ambiental les permite colonizar pacientes sin causar enfermedad, o contaminar los cultivos en el propio laboratorio.
La literatura médica nacional es escasísima acerca de esta patología. Al presente carecemos de datos confiables sobre la frecuencia de esta micosis en la República Argentina. De un estudio que integra los datos de patología fúngica generados por un número importante de laboratorios distribuidos a lo largo de todo el país, surge que el porcentaje de muestras derivadas para diagnóstico micológico es bajo. Entre enero y diciembre de 2004, tan sólo $0,78 \%$ de los casos correspondieron a zigomicosis lo que probablemente no refleja la incidencia de esta patología en la Argentina (4).

Por otra parte, el diagnóstico de especie hasta ahora no parecía tener mayor trascendencia clínica; sin embargo, existen diferencias significativas de susceptibilidad in vitro de los distintos géneros y especies de la familia Mucoraceae a los azoles, lo que podría traducirse dentro de los próximos años en importantes decisiones terapéuticas $(6,30)$.

Como todas las especies de Mucorales presentan hifas anchas no septadas que se ramifican en ángulo recto, y además causan invasión vascular, para su identificación es necesario realizar un diagnóstico morfológico en cultivos.

El diagnóstico serológico no es útil para detectar infecciones por Zygomycetes. Éstos comparten varios determinantes antigénicos, lo que dificulta la distinción entre especies e incluso entre géneros (32).

Las técnicas moleculares para la detección de zigomicosis invasora son escasas y se emplean fundamentalmente para determinar asignaciones taxonómicas. Aunque estas herramientas pueden ser útiles en los estudios epidemiológicos de brotes de zigomicosis, se conoce mal su rendimiento en el diagnóstico primario (10, 11).

En el caso clínico que presentamos concurrieron varios factores de riesgo para el desarrollo de zigomicosis cutánea: leucemia, neutropenia severa, infección bacteriana mixta y tratamiento inmunosupresor. Consideramos, junto a Chandra (3), del Palacio (5) y Runco (25) que en estos casos los pacientes presentan una evolución rapidísima y generalmente fulminante.

Habitualmente, la imposibilidad de llegar a un diagnóstico que permita el tratamiento oportuno de esta patología, induce a la medicación empírica del paciente lo que aumenta, innecesariamente, su situación de riesgo. En estos últimos años se ha descrito un incremento importante en la incidencia de esta enfermedad en instituciones aisladas o unidades específicas alcanzando un $8 \%$ en pacientes con leucemia. Este aumento del número de casos se produce generalmente en pacientes y unidades donde se administra con exceso profilaxis antibacteriana de amplio espectro, sin la sospecha de una micosis (33). 


\section{CONCLUSIONES}

La triada constituida por necrosis cutánea, gran agresividad y rápida evolución, principalmente en pacientes inmunocomprometidos, debe poner al médico en alerta sobre una posible patología fúngica. La sospecha clínica y los criterios de infección fúngica probable o posible son determinantes para establecer el diagnóstico apropiado, aún frente a la negatividad del examen directo y los cultivos. La observación de abundantes hifas hialinas no tabicadas y el aislamiento de un zygomycete deben ser una voz de alarma cuando se trata de enfermos inmunocomprometidos. La demora en el diagnóstico produce un impacto desfavorable en la toma de decisiones terapéuticas acertadas.

\section{AGRADECIMIENTOS}

Los autores agradecen al Dr. Eduardo Piontelli por haber realizado la identificación de R.oryzae y por sus sugerencias en la elaboración de este manuscrito.

\section{REFERENCIAS}

1. Amin, S. B.; Ryan, R.; Metlay, L.; Watson, W. (1998) Absidia corymbifera infections in neonates. Clin. Infect. Dis. 26: 900-902

2. Baker, R. (1957). Mucormycosis-a new disease? Jama 163:805

3. Chandra, S. \& Woodgyer, A. (2002). Primary cutaneous zygomycosis due to Mucor circinelloides. Austral. J. Dermatol. 43:39-42

4. Davel, G. \& Canteros, C. E. (2007). Situación de las micosis en la República Argentina. Rev. Arg. de Microbiología 39:28-33

5. Del Palacio, A.; Ramos, M.; Pérez, A.; Arribi, A.; Amondarain, I.; Alonso, S.; Ortiz, M. (1999). Zigomicosis. A propósito de cinco casos. Rev. Iberoam. Micol. 16:50-56

6. Diekema, D.; Messer, S.; Jones, R. \& Pfaller, M. (2003) Activities of caspofungin, posaconazol, ravuconazol, voriconazol and amphotericin B against 448 recent clinical isolates of filamentous fungi. J. Clin. Microbiol. 41:3623-6

7. Elgart, M. (1996). Zygomycosis. Derm. Clinics. 14:141-146

8. González Morán, A.; Ramos Nieto, M.; Martín López, R.; Ibáñez Pérez, R. (2007). Mucormicosis Cutánea Primaria (Rhizopus microsporus) en paciente con Leucemia Linfoide Crónica B. $9^{\circ}$ Congreso Virtual Hispanoam Anatomía Patológica_http:// www.conganat.org $/ 9$ congreso/vistaImpresion.asp?id_trabajo= 674 \&tipo $=2 /$

9. Holzel, H.; MacQueen, S.; MacDonald, A.; Alexander, S.; Campbell, C.; Warnock, D. (1998). Rhizopus microsporus in wooden tongue depressors: a major threat or minor inconvenience? J. Hosp. Infect. 38:113-8
10. Iwen, P.; Sigler, L.; Noel, R. \& Freifeld, A. (2007). Mucor circinelloides identified by molecular methods as a cause of primary cutaneous zygomycosis. J. Clin. Microbiol. 45:636-640

11. Iwen, P.; Freifeld, A.; Sigler, L. \& Tarantolo, S. (2005). Molecular identification of Rhizomucor pusillus as a cause of sinusorbital zygomycosis in a patient with acute myelogenous leukemia. J. Clin. Microbiol. 43:5819-5821

12. Kapadia, S. \& Polenkovic, H. (2004). Cutaneous Zigomicosis fdloving attempted rada atery canuation www.medscape.com. viewarticle/4957

13. Kontoyiannis, D.; Wessel, V.; Bodey, G. \& Rolston, K. (2000). Zygomycosis in the 1990s in a tertiary-care cancer center. Clin. Infect. Dis. 30:851-856

14. Kwon_Chung, K. J. \& Bennett, J. E. (1992). Mucormycosis (Phycomycosis, Zygomycosis) En: Medical Mycology. Editorial Lea and Febiger. pp.524-559

15. Lenane, P.; Keane, C. \& Loughlin, S. (2003). Mucormycosis infection presenting as a non-healing ulcer in an immunocompromised patient. Clin. Exp. Dermatol. 28:157-159

16. Nosari, A.; Oreste, P.; Montillo, M.; Carrafiello, G.; Draisci, M.; Muti, G.; Molteni, A.; Morra, E. (2000). Mucormycosis in hematologic malignancies: an emerging fungal infection. Hematologica 85:1068-1071

17. Oh, D. \& Notrica, D. (2002). Primary cutaneous mucormycosis in infants and neonates: Case report and review of the literature. J. Pediatr. Surg. 37:1607-11

18. Pagano, L.; Ricci, P.; Tonso, A.; Nosari, A.; Cudillo, L.; Montillo, M.; Cenacchi, A.; Pacilli, L.; Fabbiano, F.; Del Favero, A. (1997). Mucormycosis in patients with haematological malignancies: a retrospective clinical study of 37 cases. British J. Haematology. 99:331-336

19. Pérez-Uribe, A.; Molina de Soschin, D. \& Arenas, R. (2005). Mucormicosis cutánea primaria en un paciente con virus de la inmunodeficiencia humana. Rev. Iberoam. Micol. 22:118121

20. Ribes, J.; Vanover-Sams, C.; Baker, D. (2000). Zygomycetes in human disease. Clin. Microbiol. Rev. 13:236-301

21. Rinaldi, G. M. (1989). Zygomycosis. Inf. Dis. Clin. North Am. 3:19-41

22. Rippon, J. W. (1988). Zygomycosis. En: Medical Micology (3rd ed). Philadelphia, Saunders Co. 681-713

23. Robertson, A. F.; Joshi, V.; Ellision, D.; Cedars, J. (1997). Zygomycosis in neonates. Pediatr. Infect. Dis. J. 16:812-5

24. Roden, M.; Zaoutis, T.; Buchanan, W.; Knudsen, T.; Sarkisova, T.; Schaufele, R.; Sein, M.; Sein, T.; Chiou, C.; Chu, J.; Kontoyiannis, D.; Walsh, T. (2005). Epidemiology and outcome of zygomycosis: A review of 929 reported cases. Clin. Infect. Dis. 41:634-653

25. Runco, R.; Salim, R. \& Boente, M. (1998). Zigomicosis rino-sinuso-orbital post-traumática. Boletín Micológico 13:1115 
26. Scheffler, E.; Miller, G. \& Classen, D. (2003). Zygomycosis infection of the neonate upper extremity. J. Pediatr. Surg. 38: E21

27. Schipper, N.A.A. (1984). A revision of the genus Rhizopus I. The Rhizopus stolonifer group and Rhizopus oryzae. Studies in Mycology 25:1-19

28. Spalloni, M.; Chavez, A.; Aviles C. \& Cofré, J. (2004). Mucormicosis en pediatría. Rev. Chil. Infectol. 21:17-25

29. Sugar, A. M. (2000). Chapter 249: Agents of mucormycosis and related species. En Mandell G L, Bennett J E and Dolin R editors, Mandell, Douglas and Bennett's Principles and Practice of Infectious Diseases. Fifth edition. Churchill Livingstone, Philadelphia; 2685-2694

30. Sun, Q.; Fothergill, A.; McCarthy, D.; Rinaldi, M.; Graybill, J. (2002). In vitro activities of posaconazole, itraconazole, voriconazole, amphotericin B, and fluconazole against 37 clinical isolates of zygomycetes. Antimicrob Agents Chemother 46:1581-1582

31. Toro, C.; del Palacio, A.; Alvarez, C.; Rodríguez-Peralto, J.; Carabias, E.; Cuétara, M.; Carpintero, Y.; Gómez, C. (1998). Zigomicosis cutánea por Rhizopus arrhizus en herida quirúrgica. Rev. Iberoam. Micol. 15:94-96

32. Torres-Narbona, M.; Guinea, J.; Muñoz, P. \& Bouza, E. (2007). Zigomicetos y zigomicosis en la era de las nuevas terapias antifúngicas. Rev. Esp. Quimioterap. 20:375-386
33. Torres-Narbona, M.; Guinea, J. \& Martínez-Alarcón, J. (2007). Impact of zygomycosis on microbiology workload: A survey study in Spain. J. Clin. Microbiol. 45:2051-2053

34. Vázquez, J. (2005). Zygomycosis. http://www.emedicine.com/ cgi-bin/foxweb.exe/

35. Walsh, T. \& Pizzo, P. A. (1988). Nosocomial Fungal Infections: A classification for hospital-acquired fungal infections and mycoses arising from endogenous flora or reactivation. Ann. Rev. Microbiol. 42:517

36. Weems, J. J.; Davis, B.; Tablan, O.; Kaufman, L.; Martone, W. J. (1987). Construction activity: an independent risk factor for invasive aspergillosis and zygomycosis in patients with hematologic malignancy. Infect. Control. 8:71-75

37. Wiedermann, B. L. (1998). Zygomycosis. En: Textbook of Pediatric Infectious Diseases, Ralph D. Feigin _ James D. Cherry editors. $4^{\text {th }}$ edition. W.B.Saunders Company, Phhiladelphia. pp: 2354-2360

38. Zaoutis, T. E.; Roilides, E. \& Chiou, C.C. (2007). Zygomycosis in children: A systematic review and analysis of reported. Pediatr. Infect. Dis. J. 26:723-727 